\title{
A Novel Methodology for Efficient Retrievals of Sketch Based Images using Local Binary Patterns and Generalized Gamma Mixture Models (GGMM)
}

\author{
K M Vara Prasad, Ande Prasad
}

\begin{abstract}
The enormous work in Information technology sector met towards the development of methodologies for identifying or retrieving the images based on content. However in specific conditions narration is the best suitable way to express and hence basing the views of narration sketch based images are thus developed and utilized. These sketch based images are most useful in criminal investigations. The present article portrays a methodology for retrieving such sketch based images using the statistical modeling approaches.
\end{abstract}

Keywords: statistical models, criminal applications, Sketch-based Images, experimental evaluation, content based retrievals.

\section{INTRODUCTION}

The area of Content Based Image Retrieval has given a note worthy stride in the area of image retrievals and the main advantage with this mechanism is that based on content effective retrievals from massive datasets are made possible. This content can be either the visual features ,textual features, shape features or any other feature that helps in proper identification of image under studying and thereby helping towards efficient retrievals[1][2][3][4][5].With this features many applications have been developed and are being utilized in real time situations including the domains of health care, medical, E-business, etc., [6][7][8]. Many models are thus subjected to using both parametric model based approaches and non parametric model based approaches. however in spite of the vast advantages of the Content Based retrieval mechanisms in particular situations where individual fail to poses a query because of the ignorance of expression, effective retrievals cannot be possible. Therefore in such situation the concept Content Based Image Retrieval will fail not only this situation but also in most realistic scenarios where a witness at the crime incident tries to narrate the crime incident needs to have a more different approach. Therefore to aid these situations sketch based images came into existence [9][10][11][12] and lot of applications are thus

Revised Manuscript Received on January 22, 2020.

*Correspondence Author

K M Vara Prasad, Research Scholar, Department of Computer Science, Vikrama Simhapuri University, Nellore. Email: varaprasad.kmv@gmail.com

Ande Prasad, Professor, Department of Computer Science, Vikrama Simhapuri University, Nellore. Email: prasadjkc@yahoo.co.in

(c) The Authors. Published by Blue Eyes Intelligence Engineering and Sciences Publication (BEIESP). This is an open access article under the CC BY-NC-ND license (http://creativecommons.org/licenses/by-nc-nd/4.0/) diverted towards the usage of this application. However there is no methodology available in the literature that can identify the varied styles of the artist because of the fact that each individual poses his own articulation styles to express a particular scenario. Therefore to effectively identify such efficient scenarios statistical methodologies are well suited. Also the images so generated will have different kurtosis to understand and interpret the images with different kurtosis from GGMMs are more suited because as the skewness varies it tends to generate a new distribution, which is again a subset or a special case of gamma distribution. Therefore in this article a methodology presented considered the gamma distribution together with feature extraction based on Local Binary Patterns. The outputs derived are to be assessed for which different evaluation techniques such as precision, recall are considered. The rest of the article is highlighted in the following manner:

Section-2 provides the literature review of this paper.Section-3 highlights the dataset considered for presenting the methodology. The section-4 of the article devoted towards the feature extraction methods based on Local Binary Patterns. Section-5 of the article presents an overview of Generalized Gamma Mixture Model (GGMM) together with its special cases. In section-6 the methodologies together with experimentation is highlighted and results derived are articulated in this section of the article. The final section-7 summarizes the article.

\section{REVIEW OF LITERATURE}

In this section of the article a detailed review about various articles presented by different authors and scientists is presented to showcase the status of a present work.

Ravi Kiran Sarvadevabhatla et al.,(2017) published an article in which the authors are developed a method for rich descriptions poorly drawn sketches using multi-task hierarchical deep networks, called SketchParse. According to the authors it is first deep-network for fully automatic parsing of free hand object sketches. In this article the author's two-level fully convolutional network for sketchparse. First level is common for all object categories and second level is for expert sub-networks. This method uses a router layer to transfer the parse from shared layer to be correct expert object category layer.

Published By:

Blue Eyes Intelligence Engineering \& Sciences Publication

(C) Copyright: All rights reserved. 
This method used sketchified dataset for training databases of images. In this work the authors used canny edge detector is used to produce very important edges of image part. In this paper the authors used the datasets like TU-Berlin and Sketchy. In this work the authors prepared segmented semantic parts of databases by using PASCAL-Parts and CORE (Cross-category Object Recognition).This work outperforms the state-of-the-art CNN methods.

T Bui et al.,(2017) published a paper in which the authors are proposed a method compact descriptors for Sketch Based Image Retrieval using triplet loss Convolutional Neural Network. In this method the authors treat the SBIR problem as cross-domain modeling problem. In this article the authors used invariant pictures of sketch and photo data is learned by regression using Siamese CNN architecture with half shared weights and a triplet loss function. This learned model generalizes the categories image data. This form the basis for general cross-category SBIR. This learned data is used for indexing. After using all the above mentioned things into a unified objective function, and finally used PCA projection to obtain compact representation for sketches. In this work the authors used positive and negative exemplars with the anchor. In this paper the authors used the databases TU-Berlin and Flickr15K as a benchmark dataset. This work provides better results compared to existing learning methods.

Chunlei Peng et al., (2017) published article in which the authors have proposed the super pixel-based face sketch-photo synthesis using segmentation. According to the authors, the face detection is a difficult task since the existing methods does not perform on inherent structures. The authors proposed a novel method for face sketch-photo synthesis and this method face images are first segmented in to superpixels. These superpixels are expanded to improve compatibility of neighboring superpixels.In this method each input face image is converted to a specific graphical structure modeled by Markov networks. In this article the authors used Simple Linear Iterative Clustering(SLIC) algorithm for face segmentation. The authors used two stage synthesis process is used to learn the face structure.In this system the authors used three face sketch databases: CUFS, CUFSF, and IIIT-D.In this method a training data set is prepared with sketch-photo based on face structure as a shape. The input face image is compared with training data using Euclidian distance. The derived system performs better compared to authors developed a novel discriminative-generative hybrid model using generative task of cross-domain image composition. This model is proposed for Fine-Grained Sketch-Based Image Retrieval(FG-SBIR).In this article the authors preserved the learned embedding space, which contains domain- invariant information. This information is used for cross-domain reconstruction and used to reduce the semantic gap between image and sketch. In this article, a model takes an image as input and produces a secret code embedded via the encoder, which is used by the decoder as input to produce an image that shares the semantic information. This model uses the Sketchy Data set and QMUL-Shoe-Bag for practical implementations. This model improves the performance by adding decoder compared with the state-of-the-art methods.

Kaiyue Pang et al.,(2017) published an article in which the

existing models GoogLeNet and VGGNet.

Hua Zhang et al.,(2017) published a paper in which the authors are proposed a method for sketch classification with web images, which is called SketchNet. In this article the authors used a deep convolutional neural network to develop SketchNet. In this method the authors initially developed a triplet composed of sketch, positive and negative real image as input to the neural network. In this article the authors used softmax as a loss function to discover coherent visual structures. After that a ranking mechanism is used to make positive pairs obtain higher score compared to negative ones. After using all the above mentioned things into a unified objective function, and create feature representation for sketches. In this work the authors used three sub networks. These are R-Net for features of real images, S-Net for sketch images, and C-net for discovering the common structures for sketch and real images. In this paper the authors are used the database of TU-Berlin sketch dataset. This work provides better classification compared to the GoogLenet and VGGnet.

Andrea Manno-Kovacs et al., (2016) published article in which the authors have tried to retrieve content based images using salient orientation histograms. According to the authors, Existing sketch based image retrieval systems less effective on real life images because background data may distort image descriptors and retrieval result. In this article the authors have considered to build the descriptor only on most relevant pixels. In this work they have added preprocessing step to distinguish foreground and background, which increases importance of saliency detection. In this article a Modified Harries for Edges and Corners (MHEC) is proposed for image segmentation and $\mathrm{SOH}$ (Salient Orientation Histogram) for improved object description . Apart from that edge map is also adapted for segmentation. The derived result showcased good retrieval accuracy with minimum timestamp.

Xinghui Dong et al., (2016) published article in which the authors have proposed contours are very important features for human perceptual higher order similarity. According to the authors, human visual system can recognize only longer range contour characteristics in images. In this article the authors have examined 51 computational feature sets for human texture similarity, but no one performed well. In this paper, they are considered contour data apart from two categories of texture data :2nd order statistics and Higher Order Statistics(HOS) . In this work they have used Pertex database of 334 textures. SHOG features are considered for SBIR, which is one of its applications. Nearest-neighbour classifier is used for Natural scene recognition. The derived feature set PMIF performs well compared to existing feature sets.

Aishwarya Deore et al., (2016) published an article in which the authors have generated an advanced Sketch Based image Retrieval system using Object Boundary Selection algorithm. According to the authors, it is a complex task to reduce the gap between input sketch and photo-realistic images. 
This gap is due to noise edges in the database images. In this article the authors have proposed line segment-based descriptor called as Histogram of Line relationship (HLR). In this work they have used a noise reduction algorithm called object boundary selection. Canny edge detection algorithm is used to generate sketch from image. Apart from that HLR and boundary selection, Bag-of-visual-words(BoVW) model is used. The proposed method provides better results.

Jifei Song et al.,(2016) published an article in which the authors are proposed a method deep spatial-semantic attention for Fine-Grained Sketch Based Image Retrieval using Convolutional Neural Network. In this method the authors used attention module for spatial position of image details. In this article the authors mixed coarse and fine semantic information through a shortcut connection fusion block. This method uses a loss function to discover similar visual structures. The matching mechanism is performed by using the higher order learnable energy function (HOLEF) based loss. This method used attention module and coarse-fine fusion block to fuse multiple layers at different levels. After using all the above mentioned things into a unified objective function, and create feature representation for sketches. In this work the authors used Mahalanobis distance metric used in loss function. In this paper the authors are used the datasets like QMUL-Shoe and QMUL-Chair and Handbag a benchmark. This work outperforms the existing methods.

Yonggang Qi et al.,(2016) published a paper in which the authors are proposed a method for Sketch Based Image Retrieval using Siamese Convolutional Neural Network. In this method the authors initially developed output feature vectors closer for input sketch-image pairs and eliminated irrelevant images.In this article the authors used a loss function to discover similar visual structures. The ranking mechanism is used to make positive pairs obtain higher score compared to negative ones. After using all the above mentioned things into a unified objective function, and create feature representation for sketches. In this work the authors used Stochastic Gradient Descent(SGD) for the learning of Siamese CNN. In this paper the authors are used the database Flickr15k as a benchmark dataset. This work provides better results compared to non learning methods.

Lihua Tian et al.,(2016) published an article in which the authors generated a new approach for image segmentation using multi-layer clustering algorithm, In this article the authors proposed two stage clustering. In the first stage mean shift algorithm is used to divide the images into smaller domain and in the second stage hierarchical clustering is used. This algorithm is called MSHC(Mean Shift Hierarchical Clustering).In this article the authors used Ward algorithm as the Hierarchical clustering algorithm. The authors collected all images from Berkeley's BSDS500. All the tests are conducted in MatLab. This multi-layer clustering approach provides better results compared to the Mean shift and K-means algorithms.

Shantanu Deshpande et al.,(2016) published a paper in which the authors are proposed an alternative method for Sketch Based Image Retrieval which uses Siamese and Triplet network. In this article the authors used a deep residual learning network. In this method the authors initially developed a triplet composed of sketch, positive and negative real image as input to the residual network. In this article the authors used a loss function to discover coherent visual structures. The ranking mechanism is used to make positive pairs obtain higher score compared to negative ones. After using all the above mentioned things into a unified objective function, and create feature representation for sketches. In this work the authors used three sub networks for discovering the common structures for sketch and real images. In this paper the authors are used the database of Sketchy dataset. This work provides better results compared to the GoogLenet.

\section{DATA SET CONSIDERED}

In order to present the proposed methodology, a real time data set is developed and is utilized for the experimentation purpose. Every image is preprocessed and it is normalized to a fixed size of 150X150.these image comprises of sketch views generated by different artist with different strokes and curvatures. The sample data set is depicted in Fig.1 of the article.

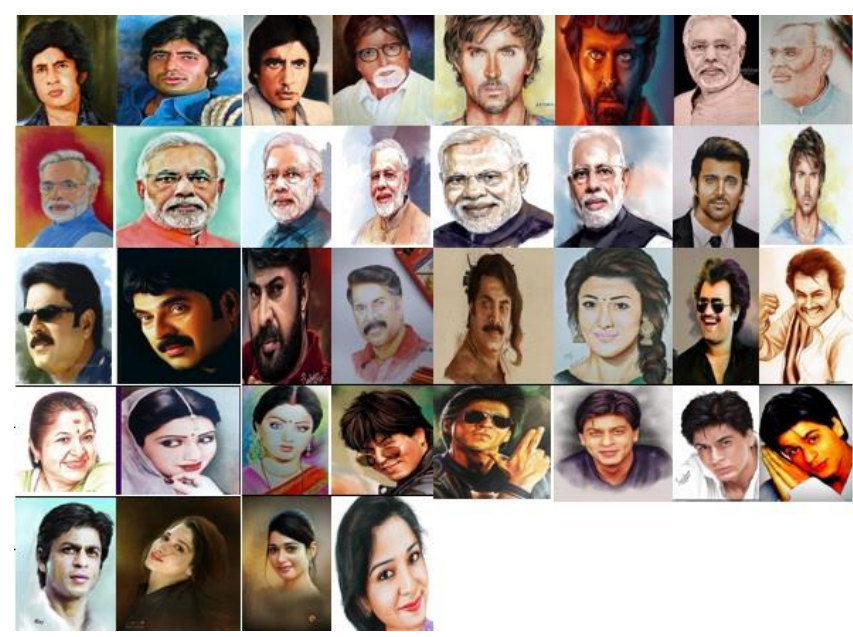

Fig. 1.Sample data set.

\section{LOCAL BINARY PATTERNS}

Every artist exhibits the skill with different stroke variations and also expressed his view of thought in different styles and in different forms. Therefore to have the exact inference about the school of thought of artist aspect the images articulated. The semantic meaning should also be considered. With this thought process in this article, Local Binary Patterns are considered together with correlation coefficient for which the semantic interpretation can be underlined.

The main advantage behind the usage of Local Binary Patterns is that it can identify a feature posed by the individual as a query and can interpret the images having different orientations. in the adjacent images, local pattern in LBP operators describes the relationships between the pixel and the adjacent pixels. Based on LBP operator, local samples are presented by binary codes that are composed of a threshold of gray values neighborhood according to the relevant value of central pixels. The Fig. 2 specifies the LBP operation.

Published By:

Blue Eyes Intelligence Engineering \& Sciences Publication

(C) Copyright: All rights reserved. 


\begin{tabular}{|c|c|c|}
\hline 70 & 57 & 20 \\
\hline 50 & 35 & 5 \\
\hline 68 & 39 & 48 \\
\cline { 1 - 1 } & \multirow{2}{*}{35} \\
\hline
\end{tabular}

Fig. 2. Basic LBP Operation.

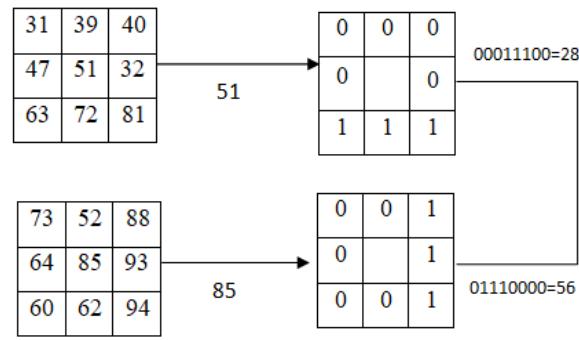

Fig. 3. LBP implementation for single pixel.

The vector of the LBP function is created in its simplest form as follows:

1. Divide the image block into cells (for example, 16x16 pixels for each cell).

2 . For each pixel in a cell, compare the pixel with each of its 8 neighbors (upper left, middle left, lower left, upper right, etc.). Follow the points along the circle, that is, clockwise.

3. If the pixel value of the medium is greater than its neighbor, mark it as "0". Otherwise, mark as "1". This generates an 8-digit binary number (which usually becomes a decimal number for convenience).

4. Through the cell, a histogram of the frequency of each "number" that occurs (ie, any combination of whose points are smaller and larger than the center) is calculated.

5. Optionally, normalize the histogram. Link the histograms (normalized) of all cells. This provides a vector of features for the entire window.

The function vector is now given as input to the classifier based on GGMM.

\section{GENERALIZED GAMMA DISTRIBUTION}

The probability density function of generalized gamma distribution is given by

$$
f(x, k, c, a, b)=\frac{c(x-a)^{c k-1} e^{-\left(\frac{x-a}{b}\right)^{c}}}{b^{c k} \Gamma(k)}
$$

Where a, b, c, k is called the Gamma variants and c, k is called shape parameters such that $\mathrm{c}, \mathrm{k}>0$. a is called location parameter, $\mathrm{b}$ is called shape parameter with $\mathrm{a}, \mathrm{b}>0$.

The mean of the Generalized Gamma Distribution is given by

$$
\frac{a+b \Gamma\left(\mathrm{c}+\frac{1}{\mathrm{k}}\right)}{\Gamma(\mathrm{c})}
$$

The variance of the Generalized Gamma Distribution is given by

$$
\left.b^{2} \frac{\Gamma\left(\mathrm{c}+\frac{2}{\mathrm{k}}\right)}{\Gamma(\mathrm{c})}-\left\{\Gamma\left(\mathrm{c}+\frac{1}{\mathrm{k}}\right) / \Gamma(\mathrm{c})\right]^{2}\right\}
$$

The mode of Generalized Gamma Distribution is given by

$$
a+b\left(c-\frac{1}{k}\right)^{\frac{1}{k}, c}>\frac{1}{k}
$$

The rth moment about the Location parameter ' $a$ ' is given by

$$
b^{k}(\Gamma(\mathrm{c}+\mathrm{r} / \mathrm{k}) /(\Gamma(\mathrm{c}))
$$

We assume that every image as a k-component Generalized Gamma distribution and its Probability Density Function is of the form

$$
h(x)=\sum_{i=1}^{k} \alpha_{i} g_{i}\left(y_{i} \mid \mu_{i}, \sigma_{i}^{2}, \lambda\right)
$$

Here, $\lambda$ is the skewness parameter and $\mathrm{k}$ is the number of regions, $\alpha_{i}>0$ are weights such that $\sum_{i=1}^{k} \alpha_{i}=1$ and

$$
f(x, k, c, a, b)=\frac{c(x-a)^{c k-1} e^{-\left(\frac{x-a}{b}\right)^{c}}}{b^{c k} \Gamma(k)}
$$

$\alpha \_i$ is the probability of occurrence of the ith component of the Generalized Gamma Distribution, i.e., the probability of the ith image sample. Generally, it can be taken as the ratio of the size of the ith pixel to the size of the entire image data such that,

$$
\sum_{i=1}^{k} \alpha_{i}=1
$$

\section{EXPERMENTATION AND RESULTS}

The experimentation is conducted in Dot NET environment considering the real-time generated dataset containing 1000 different sketch based images, for the experimentation with Dataset. Among this dataset we have considered 100 sketches data as test data.

In order to experiment the model proposed, we have considered the data set and the features are extracted using Local Binary Patterns and the classification is carried out using GGMM. The various inputs and outputs are as follows:

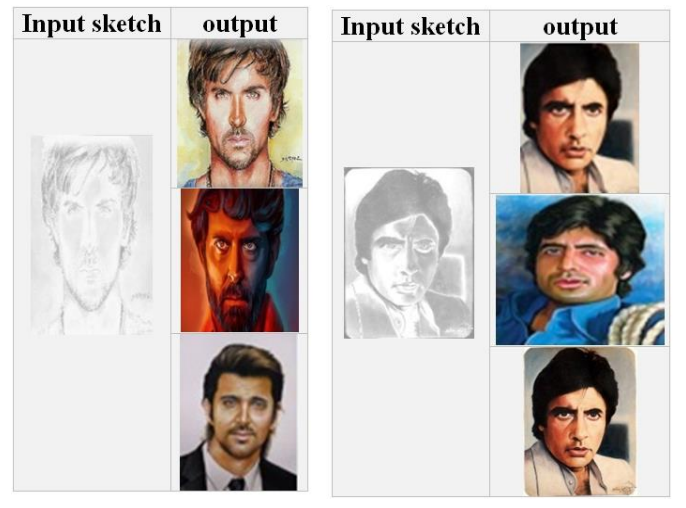

Fig. 4. Input and Output images.

In order to evaluate the model various performance metrics such as Precision, Recall, Error Rate and Retieval Efficiency are used. DCT is used as a feature vector for all the metrics. The following table shows various performance metrics for real-time dataset.

Table- I: Performance Metrics

\begin{tabular}{|c|c|c|c|c|c|c|}
\hline $\mathbf{R}$ & NR & R/NR & Precision & Recall & $\begin{array}{c}\text { Error } \\
\text { Rate }\end{array}$ & $\begin{array}{c}\text { Retrieval } \\
\text { Efficiency }\end{array}$ \\
\hline 80 & 500 & 0.16 & 80 & 6 & 20 & 80 \\
\hline 70 & 500 & 0.14 & 74 & 26 & 26 & 74 \\
\hline 60 & 500 & 0.12 & 62 & 35 & 38 & 62 \\
\hline 50 & 500 & 0.1 & 53 & 42 & 47 & 53 \\
\hline 40 & 500 & 0.08 & 42 & 53 & 58 & 50 \\
\hline 30 & 500 & 0.06 & 35 & 62 & 65 & 46 \\
\hline 20 & 500 & 0.04 & 26 & 74 & 74 & 45 \\
\hline
\end{tabular}

$R:$ No. of relevant to sketch based images in the database.

NR: No. of non-relevant to sketch based images in the database. $R / N R$ : Ratio of relevance to non-relevance.

Published By:

Blue Eyes Intelligence Engineering \& Sciences Publication

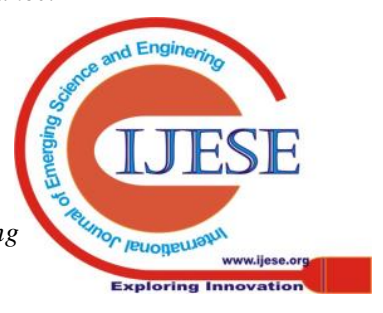


The graphical representation of the performance metrics is shown in below figure.

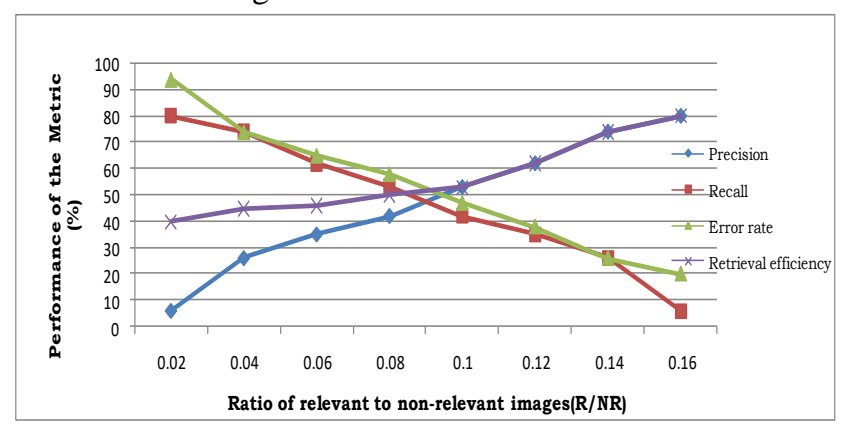

Fig. 5. Comparision of all the metrics.

\section{CONCLUSION}

In this article a methodology is presented to retrieve the image based on sketches. In order to extract the features, local binary patterns is considered. The Query image is posed and based on the relevance, most relevant to sketch based images are extracted. The classification is carried out using GGMM algorithm. The performance accuracy is carried out using various metrics like Precision, Recall, Error Rate and Retrieval Efficiency and the results are presented in Table 1. The results developed showcase that the developed methodology could provide better retrieval accuracy. This method can be well suited for application of cyber forensic.

\section{REFERENCES}

1. G. Ravi Kiran Sarvadevabhatla,Isht Dwivedi,Abhijat Biswas,Sahil Manocha and R.Venkatesh Babu," SketchParse : Towards Rich Descriptions for Poorly Drawn Sketches using Multi-Task Hierarchical Deep Networks",IEEE,2017.

2. T.Bui,L.Ribeiro, M.Ponti and J. Collomosse," compact descriptors for sketch-based image retrieval using a triplet loss convolutional neural network",CVIU,2017.

3. [Chunlei Peng, Xinbo Gao, Nannan Wang,and JieLi," Superpixel-Based Face Sketch-Photo Synthesis",IEEE,2017.

4. Kaiyue Pang, Yi-Zhe Song, Tao Xiang, and Timothy M. Hospedales,"Cross-domain Generative Learning for Fine-Grained Sketch-Based Image Retrieval",2017.

5. Hua Zhang, Si Liu,Changqing Zhang, wenqi Ren, Rui Wang,and Xiaochum Cao,"SketchNet:Sketch Classification with Images",IEEE,2016.

6. Andrea Manno-Kovacs," CONTENT BASED IMAGE RETRIEVAL USING SALIENT ORIENTATION HISTOGRAMS",ICIP,2016.

7. Xinghui Dong and Mike J. Chantler,"Perceptually Motivated Image FeaturesUsing Contours",IEEE TRANSACTIONS ON IMAGE PROCESSING, VOL. 25, NO. 11,2016.

8. Aishwarya Deore, Dr.B.L. Gunjal, "A Survey On Sketch Based Image Retrieval Technique",IJARIIE,2016.

9. Jifei Song,Qian Yu,Yi-Zhe Song, Tao Xiang and Timothy $\mathrm{M}$. Hospedales," Deep Spatial-Semantic Attention for Fine-Grained Sketch-Based Image Retrieval",

10. Yonggang Qi,Yi-Zhe Song,Honggang Zhang and Jun Liu, SKETCH-BASED IMAGE RETRIEVAL VIA SIAMESE CONVOLUTIONAL NEURAL NETWORK",IEEE,2016.

11. Lihua Tian, Liguo Han, and Junhua Yue," Research on Image Segmentation based on Clustering Algorithm",IJSP,2016.

12. Shatanu Deshpande, and Naman Goyal,"Sketch Based Image Retrieval",IEEE,2016.

\section{AUTHORS PROFILE}

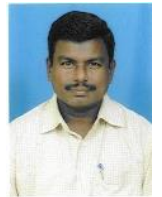

Mr. K. M. Vara Prasad, had obtained his B.Tech and M.Tech in Computer Science and Engineering from Acharya Nagarjuna University, Guntur, Andhra Pradesh, India. He has teaching experience of 10 Years in the department of Computer Science and Engineering. Currently he is perusing Ph.D in Digital Image Processing.

Published By:

Blue Eyes Intelligence Engineering \& Sciences Publication (C) Copyright: All rights reserved. Cloud Computing and Computer Networks etc.

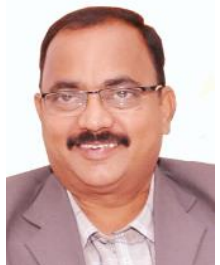
viz Co-ordinator, Head, Vice-Principal, Chairmen and Member (Ut Board of Studies in Computer Science / Computer Science and Engineering, Officer In-charge: College Information Cell, Member: Technical Advisory Committee etc. He is as Supervisor/ Co-supervisor to guide Ph.D.'s and is currently guiding six Ph.D. Full-Time and Part-Time scholars. He has more than 50 publications in International and National Journals and Conferences with good impact factor and reckoning. His research insights were presented in Conferences, Seminars and Symposia both on National and on the International platforms. His research areas of interests are Speech Processing, Pattern Recognition, Image Processing,

Dr. Ande Prasad, received his M.Sc. (Mathematics), (Computer Science \& Technology), Ph.D. (Compute Science \& Systems Engineering) from Andhra University, Visakhapatnam, Andhra Pradesh, India. $\mathrm{He}$ is working as professor in computer science , vikrama simhapuri university and more than 23 years of enriched experience in teaching, research and administration. He worked in various

His research areas of interest are Digital Image Processing, Artificial (1) 